\title{
Metamorfosis del espacio expositivo en el museo de ciencias: de cueva de tesoros a estudio creativo
}

\section{Resumen}

La ciencia se ha entendido como la evidencia de la racionalidad de la cultura occidental y el museo de ciencias como el espacio donde esa imagen de racionalidad es expuesta públicamente. Desde la concepción del museo como institución pública hasta hoy, los imaginarios culturales de la ciencia han evolucionado y en consecuencia los espacios para su representación. Por ello el siguiente análisis toma como unidad de estudio el espacio expositivo puesto que, al converger en él tanto contenido como audiencia, es el lugar en el cual se compone el imaginario sobre lo expuesto ya sea por lo que se propone desde su diseño o por la interpretación que se obtiene al interactuar en este. Se distinguirán tres espacios expositivos proyectados en el museo de ciencias que, pese a que han emergido y supuestamente relevado por circunstancias y percepciones históricas del museo, hoy se sobreponen y conviven en gran parte de estas instituciones: la cueva de tesoros; la tienda por departamentos y el estudio creativo. Comprender estas tipologías permitirá distinguir el papel que juega dicha institución para la diseminación y divulgación de la ciencia a la sociedad, además de las diferentes percepciones culturales que se han inoculado de la ciencia.

\author{
Natalia Pérez-Orrego \\ PhD. en Diseño y Creación \\ Master en Arquitectura, arte y \\ espacios efímeros \\ Docente en Diseño Industrial \\ Universidad Pontificia Bolivariana \\ Medellín, Colombia \\ Correo electrónico: natalia. \\ perezorrego@upb.edu.co \\ ๑ orcid.org/0000-0001-8527-0568 \\ Google Scholar \\ John Arango Flórez \\ Magíster en Arquitectura \\ Especialista en Estudios Urbanos \\ Docente en Arquitectura Universidad \\ Nacional sede Medellín \\ Medellín, Colombia \\ Correo electrónico: jfarang3@unal. \\ edu.co \\ (ㄷ) orcid.org/0000-0002-9975-3234 \\ Google Scholar
}

Recibido: Agosto 16 de 2017 Aprobado: Septiembre 9 de 2018

\section{Palabras clave:}

Comunicación de las ciencias, espacio expositivo, museo, experiencia. 


\section{Metamorphosis of the exhibition space at the science museum: from treasures cave to ceative studio}

\begin{abstract}
Science has been understood as the evidence of the rationality of Western culture and the Science Museum as the space where that image of rationality is publicly exposed. From the conception of the museum as a public institution until today, the cultural imaginaries of Science have evolved and, consequently, spaces for their representation have also changed. Therefore, the following analysis takes the exhibition space as a unit of study since when both, content and audience, converge on it, it is the place in which the imaginary is composed on what is exposed, either by what is proposed from its design or by the interpretation obtained when interacting in it. Three exhibition spaces will be projected at the Science museum and, although they have emerged and supposedly have being replaced by different circumstances and historical perceptions of the museum, today they are superposed and coexist in a large part of these institutions: the treasure cave, the department store and the creative studio. Understanding these typologies will allow distinguishing the role played by this institution in the divulgation and dissemination of science to society, in addition to the different cultural perceptions that have been inoculated from Science.
\end{abstract}

Key words:

Science communication, exhibition space, science museums, experience. 


\section{Introducción: el museo como medio de cultura científica}

El museo de ciencias $(\mathrm{MC})^{1}$ es una de las instituciones más importantes para la divulgación científica, ya que siempre ha buscado presentar sus hallazgos con un lenguaje más espontáneo y accesible para un público diverso. Desde que se convirtió en institución pública, a principios del siglo XVIII, el museo ha utilizado diversas estrategias para ello; pero la más representativa es su espacio expositivo con el diseño de vitrinas cerradas, máquinas interactivas, juegos, laboratorios, talleres entre otros; los MC han pasado por diferentes maneras de materializar la comunicación entre el público y la ciencia. En consecuencia, estos diferentes medios de expresión han derivado en diversas comprensiones culturales de la ciencia.

Independiente del medio de comunicación utilizado, el MC ha promovido la comprensión de la cultura científica como un bien público. Durante siglos el coleccionismo estuvo celosamente cuidado bajo llave desde la primera institución helenista conocida como Museion², que hacia parte de la Biblioteca de Alejandría, hasta los templos romanos en los que su acceso estaba restringido dado que sus colecciones de objetos eran consideradas como tesoros públicos. Al coleccionismo le tomó tiempo abrir sus puertas al público y pasar de ser una colección hedonista y económica, a una con valor formativo y científico con la aparición del humanismo renacentista; a partir de ello los objetos comenzaron a ser considerados un bien público, aunque continuaban teniendo un aura aún elitista. Pero es cuando los objetos coleccionados pasan a estar cobijados por una estructura de orden y clasificación con el advenimiento de la llustración y el Enciclopedismo en el siglo XVIII que se les permitió ser presentados y

\footnotetext{
${ }^{1}$ Esta institución también es denominada Centros de ciencia y tecnología o Museos para niños. Con la incursión de los medios interactivos, los centros de ciencia y tecnología quisieron diferenciarse de los museos al utilizar este tipo de mediación tecnológica; hacer esta diferencia nominal a causa de esta implementación es inútil; hoy, cualquier tipología de museo recurre al uso de este tipo de medios (Poulot, 2011). Para e efecto de esta investigación, entonces, se entenderá al museo de ciencia (MC) como el lugar que abarca las anteriores designaciones.

${ }^{2}$ El Museion fue el templo dedicado a las musas protectoras de las artes y las ciencias. Fue un lugar de estudio, de investigación, dedicado a las ciencias y la filosofía (Labandeira, 2008).
} 
exhibidos bajo discursos cientificistas, bajo una entidad que comenzaba ya a ser considerada una verdadera institución pública: el museo (Pastor, 2011).

Los museos nacidos desde el siglo XIX hasta el siglo XX tendían, en su mayoría, a ser un gran espacio de recolección; tal como lo señala Pastor (2011): "el destino del museo, en tanto espacio para el almacenamiento de una cultura fosilizada y para la contemplación silenciosa, pasiva y reverente de una realidad histórico-cultural cerrada dentro de vitrinas, que solo alcanzaban a entender unos cuantos privilegiados" (p. 29). Esa situación de coleccionismo entumecido comienza a trastocarse por las crisis financieras, educativas e industriales de la década de 1960 y que, con el suceso del mayo francés, impulsaron al museo por un cambio de apertura social y educativa hacia su comunidad; provocando ya no solo su participación como conservador, sino también como un divulgador cultural (Bellido, 2001).

El paso de la posesión del conocimiento de unos privilegiados a ser un dominio público y cultural ha volcado al museo a comprenderse no solo como una institución de educación científica no formal, sino también para el fomento de la cultura científica. Esto le permite atender sus exhibiciones bajo nuevas narrativas, ya alejadas de las que se podrían denominar narrativas enciclopédicas, hacia unas temáticas estimulantes y atractivas para el público común donde ellos vean reflejadas situaciones de su vida cotidiana y que no necesariamente deban implicar una formación intelectual profunda para su disfrute.

\section{Tres espacios, tres medios, tres mensajes}

Partiendo del conocido aforismo de Marshall McLuhan (1996) "el medio es el mensaje" se proponen aquí tres momentos, tres estadios por los que el MC ha trasegado desde sus orígenes hasta nuestros días, a partir de la denominación 
de tres espacios expositivos que son el medio y a la vez el mensaje; se expondrá la metamorfosis que ha sufrido el MC como espacio de comunicación de la ciencia al analizar tres elementos circunscritos en dicho espacio: el contenido científico presentado; los artefactos expositivos que conservan, visualizan y/o materializan el contenido y la experiencia que sostiene el público en este lugar.

\section{La cueva de tesoros: un espacio para la contemplación. El medio: la descripción. El mensaje: ¡la ciencia existe!}

Los primeros escenarios expositivos de los MC se desarrollaron en torno a grandes colecciones naturales, por lo que su espacio expositivo se vio abocado a presentar descripciones de los especímenes albergados y conservados en vitrinas que hacían de la participación del visitante un gozo contemplativo y desbordado hacia la observación. Aquel cuerpo expositivo de la ciencia consiguió ilustrar a los ciudadanos sobre el discurso racionalista y, por ende, transformarlos en testigos del resultado de la teoría científica (Bradburne, 1990); lo que cumplía así con el cometido de la Ilustración: disipar las tinieblas mediante las luces de la razón.

En este primer hecho se afirmó el interés que la ciencia concedía a los objetos que la exponían. La presentación de sus colecciones, naturales y técnicas, fueron expuestas como tesoros de estudio y catalogación taxonómica; los objetos al ser valorados como evidencias se conservan en vitrinas, estanterías y dioramas para ser protegidos del medio ambiente y del público a través del cristal; y así, el público deviene en espectador de lo presentado. Aquí la función de la puesta en escena es clara: conservar para enseñar; lo que supone una comprensión del artefacto expuesto más como el hallazgo que describe y evidencia el fenómeno o el espécimen expuesto. Esta situación conservacionista que propone el museo, y la misma ciencia, plantea una mirada de un público que acepta lo descrito y que sigue las reglas de clasificación 
taxonómica expuestas; lo que adiciona a la relación entre conocimiento y artefacto expositivo, una experiencia pasiva del visitante ante lo presentado; es un espectador que concentra su actividad en la observación, por lo que también se ha denominado a este tipo expositivo como eyes on (ojos a la obra). En síntesis, en esta configuración expositiva se distingue: una compresión del contenido científico como evidencia, al artefacto como el medio que conserva para enseñar y que dispone que la experiencia del visitante sea la de contemplar y atestiguar de manera pasiva.

La Gran Galería de la Evolución del Museo Nacional de Historia Natural de Francia, en París (Figura 1), reformada por los arquitectos y diseñadores Borja Huidobro, Pablo Chemetov y René Allio en 1994, presenta una arquitectura expositiva en estos términos: un gran espacio expositivo central donde reposan especímenes de grandes dimensiones en posturas naturalizadas y en torno a este se encuentran las pasarelas vidriadas en varios niveles que dirigen las circulaciones del público que observa pasivamente al caminar entre las colecciones. Esta es la organización estereotípica de los museos de ciencias tradicionales donde el ejercicio discursivo del espacio se concentra evidenciar la existencia de los objetos expuestos (Figura 2). 


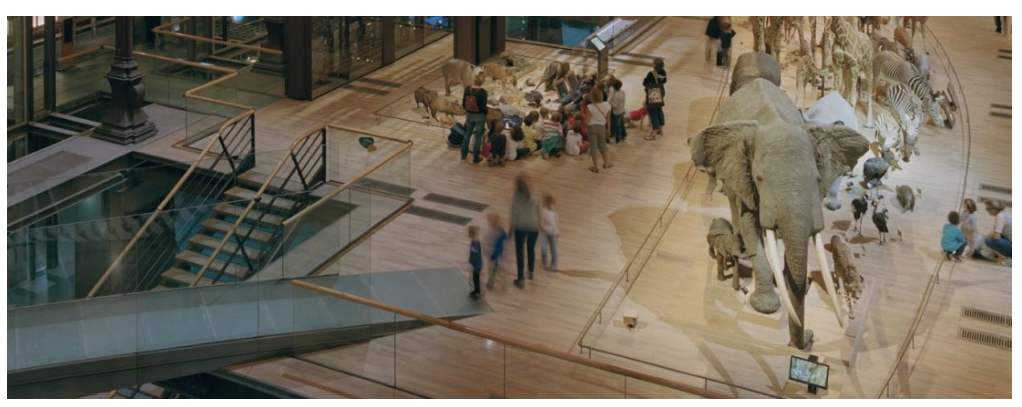

Figura 1. "Caravana africana". Museo Nacional de Historia Natural de París. Fuente: http://www.mnhn.fr/fr/visitez/activites-scolaires-extrascolaires.

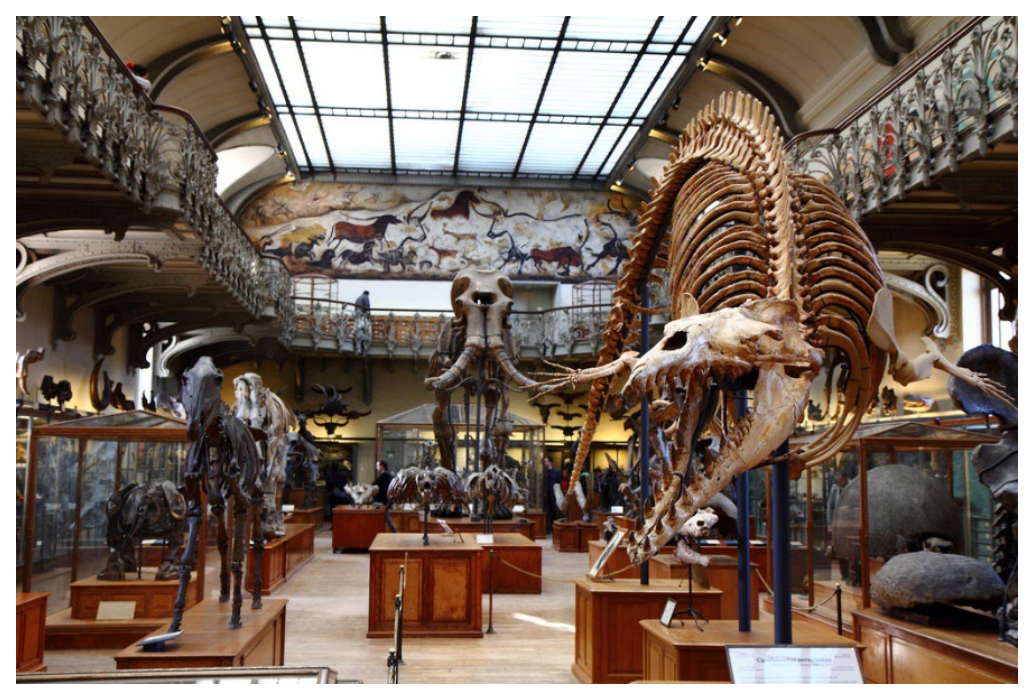

Figura 2. "Galería paleontológica". Museo Nacional de Historia Natural de París. Fuente: https://www.flickr.com/photos/holandarodrigo/8718454245. 
Ciertamente el dar testimonio sobre la ciencia y cultivar el saber racional sobre sus conceptos y teorías en los visitantes a través de la descripción procura un escenario muy útil a la ciencia para darse a conocer, pero que trae como consecuencia que el público pierda el interés al agotarse rápidamente el contendido expuesto y haciendo que la ciencia se interprete desde un racionalismo estático e incuestionable. Estas consecuencias de la exposición descriptiva motivaron la apertura de aquellas vitrinas tradicionales que encerraban las evidencias del conocimiento para permitir al público la manipulación de aquel cuerpo con el que se exponía la ciencia (Bellido, 2001; Franco-Avellaneda, 2013; Orozco, 2005; Pastor, 2011). Esa pretensión de cambio reconoció no solo concentrar la mirada en el conocimiento a divulgar, sino también en la manera en cómo este se exponía ante el público; lo que promovió la divulgación pasiva hacia una verificación activa por parte del público.

\section{La tienda por departamentos: un espacio para el consumo de la ciencia. El medio: la demostración. El mensaje: ¡la ciencia es verificable!}

Proponer la intervención activa del público provocó que el MC reexaminara su experiencia expositiva. La exposición descriptiva de los primeros MC, distinguidos también como MC de primera generación ${ }^{3}$ por la autora Paulette McManus (1992), fue ajustada a la luz de la reforma educativa de los años 60 que buscó retomar la pedagogía progresista ${ }^{4}$ de Rousseau, Pestalozzi, Fröbel, Dewey. Este movimiento rechazaba el modelo de aprendizaje basado en la memorización, ya que anulaba el carácter participativo que el aprendiz podría tener con la teoría y debilitaba así su hambre de curiosidad (Nussbaum, 2010); por el contrario, la pedagogía progresista propendía más bien por un

${ }^{3}$ Se considera al MC de primera generación, al museo tradicional que centra su exposición en la clasificación taxonómica de su colección; además de mantener un carácter conservacionista e investigativo. James Bradburne (1990) denominó a esta generación: taxonomía imperial.

${ }^{4}$ Una de sus principales bases filosóficas está planteada en el texto Emilio de Jean-Jacques Rousseau (1982) donde su principal modelo de inspiración se centra en la Mayéutica Socrática con la que el niño forma un criterio con base en la argumentación. Para ampliar, ver el capítulo, "La pedagogía socrática" del texto Sin fines de lucro de Martha Nussbaum (2010). 
aprendizaje de naturaleza práctica en la que el sujeto aprende poniendo manos a la obra. De ahí que la incursión del público en el MC, a partir de la manipulación de los contenidos, dio paso al MC de segunda generación con el fin de demostrar a través de la acción física los fenómenos científicos presentados.

Uno de los primeros museos que utilizó la acción demostrativa como discurso expositivo fue el Exploratorium de San Francisco. Frank Oppenheimer, físico y académico norteamericano, se basó en la librería de experimentos que previamente había construido para el laboratorio de física de estudiantes de primer año en la Universidad de Colorado para conformar la exposición interactiva con la que inauguró el Exploratorium en 1969. Su concepción estaba centrada en una centena de artefactos que presentaban experimentos manipulables hands on (manos a la obra), buscando promover la curiosidad y la exploración para alejarse de la descripción textual de los fenómenos que no lograban interesar a los visitantes ${ }^{5}$. Así la incursión de los artefactos para explicar la ciencia no fue la novedad de Oppenheimer, la novedad radicó en utilizarlos como un instrumento empático para divulgar las ciencias.

Esta implementación discursiva llevó a Oppenheimer (1968) a reunir estética y conocimiento en sus experimentos en busca de generar esa empatía participativa con la ciencia: "las demostraciones y exhibiciones del museo deben tener un atractivo estético, así como un propósito pedagógico, y deben ser diseñados para hacer las cosas más claras en lugar de cultivar el oscurantismo o la ciencia ficción" (p. 206).

El enunciado de Oppenheimer distingue, por primera vez, la responsabilidad que tiene el diseño del espacio expositivo frente a la divulgación de la ciencia. La estética y el propósito pedagógico son dos rasgos importantes 
para la comunicación científica en los MC, pero la conjunción de estos en exposiciones demostrativas es un resultado que se ha criticado como incipiente para la comunicación científica. Desde del lado estético, se sostiene que es un aspecto que sensibiliza la ciencia a tal punto que la convierte en un espectáculo perceptivo y la banaliza; y del lado pedagógico, se sostiene que el conocimiento que logra llevarse el visitante al manipular palancas y botones es insulso con respecto al fenómeno científico (Hernández y Rubio, 2009).

Con la aparición de las exposiciones interactivas, a finales de los años 80 en los MC de segunda generación, se incrementaron las capacidades cualitativas y cuantitativas de intervención del público frente a los programas presentados. Si bien es claro que su implementación amplió el rango de posibilidades de acercamiento al fenómeno, el MC interactivo sigue sosteniendo una exposición demostrativa; pues, a pesar de su diversificación tecnológica, este tipo de comunicación aún no permite una reciprocidad abierta entre público y ciencia. Pasar de manipular artefactos análogos que presentan una única respuesta, a participar con una serie de instrumentos y artefactos interactivos que permiten la elección entre variables para obtener una serie de respuestas relacionadas con el hecho científico no es un cambio que pueda ser homologable con una evolución sustancial del medio demostrativo; por el contario, estos artefactos que han sido denominados interactivos realmente siguen manteniendo dicho espacio expositivo. En consecuencia, estas distinciones señalan que las exposiciones interactivas no son suficientes para proponer un cambio sustancial en la comunicación en el MC.

Adicionalmente las críticas realizadas al medio demostrativo se agudizan al señalar que el MC no logra comunicar que la ciencia es una actividad humana, la cual se presenta como una actividad de resultados incuestionables y fáciles de hallar sin dar cuenta del carácter falible del científico — como ser humano- en sus intentos por lograr una integridad de los resultados (Rennie, 2007). Sobre 
estos señalamientos el arquitecto, diseñador y especialista en museos James Bradburne, en su texto "Beyond Hands-On: Truth-telling and the Doing of Science" (1990), contribuye a dichas críticas al referirse a este espacio expositivo como una tienda por departamentos en la que se exhiben los fenómenos en máquinas atractivas y en las que el visitante pasa de una a otra buscando ser sorprendido pero sin remplazar o modificar el criterio previo con el que llega al museo. Por consiguiente, Bradburne considera que este espacio sigue perpetuando la misma estructura expositiva y positivista del siglo XIX; es decir que el fenómeno presentado solo permite ser atestiguado y es restrictivo con respecto a las diversas conclusiones que el visitante pueda generar.

En todo caso hay una importante diferencia en cuanto a la experiencia que el cuerpo tiene en un MC de segunda generación, ya que involucra los sentidos más allá de la vista; la experiencia hands on pretende involucrar a todo el cuerpo, de tal manera que la relación con el conocimiento sea más dinámica. Todas estas situaciones experienciales tienen una estrecha relación con la manera en que se ha entendido la relación del cuerpo y el espacio desde la arquitectura moderna hasta hoy; en donde se ha pretendido por parte de los arquitectos involucrar toda la gama de agentes estéticos para lograr una experiencia completa e inmersiva en la actividad del espacio (Vélez, 2013), pero aun así es la vista el sentido por excelencia.

La experiencia del museo de segunda generación ha crecido muy de la mano con los avances lúdicos contemporáneos como los juegos de vídeo, que empezaron siendo simples proyecciones de una pantalla que reaccionaba a las acciones de una palanca o un botón hasta los juegos inmersivos de realidad virtual y los que involucran todo el cuerpo como Nintendo Wii, Kinect y Move. La experiencia del museo pasó así de ser meramente contemplativa (visual) a ser mas sinestésica, claro, sin olvidar que la vista sigue siendo predominante en el campo de la experiencia cognitiva museística. 
En consecuencia, el espacio expositivo del MC de segunda generación se ha concebido como un gran contenedor universal donde la exposición de los artefactos se dispone desperdigada y no hay una diferencia clara entre la circulación y la exposición; esto en virtud de la posibilidad de manipular las máquinas y objetos que se exponen; entonces el espacio expositivo aparece como una disposición aparentemente caótica de cosas con gente entre ellas. El museo Exploratorium de San Francisco, por ejemplo, conserva esta estrategia desde la configuración de su primer espacio expositivo (Figura 3) y la reproduce en las nuevas instalaciones ubicadas en el Pier 15 e inauguradas en abril de 2013 (Figura 4). Esta nueva versión del museo se localiza en una antigua bodega de la bahía de la ciudad, el espacio interior es una gran nave a dos niveles donde la percepción del público es la unidad espacial en la que se localizan pequeñas áreas contenidas en las que se ubican las máquinas y artefactos; podría decirse que casi atravesadas en la circulación para que el público se tope con cada una de ellas.

Esta disposición se puede ver en otros museos interactivos como el Museo de Ciencia e Industria de Chicago (Figura 5) donde el espacio total está poblado de microespacios con experiencias particulares y el público pasa de una a otra exposición sin interferencias, donde el espacio interior tiene además un carácter de espectacularidad en el diseño de la iluminación y el color a la manera de los casinos, lo que le concede así una percepción inmersiva hacia el contenido expuesto. 


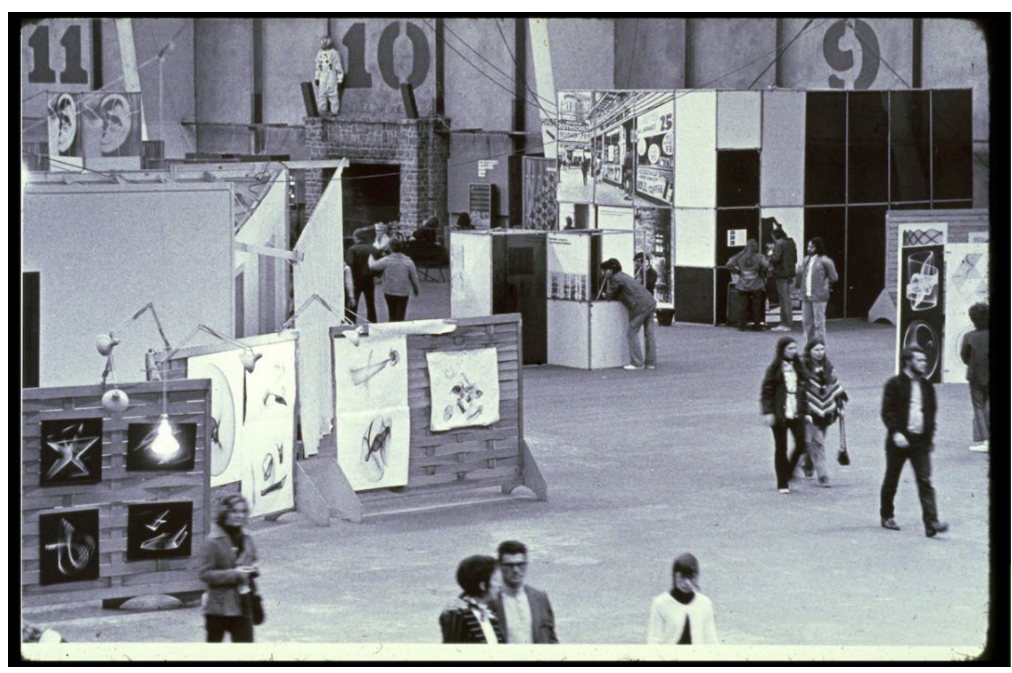

Figura 3. "Harmonographs on the museum floor, 1970's". Museo Exploratorium, San Francisco. Fuente: http://explodingtorium.tumblr.com/page/4

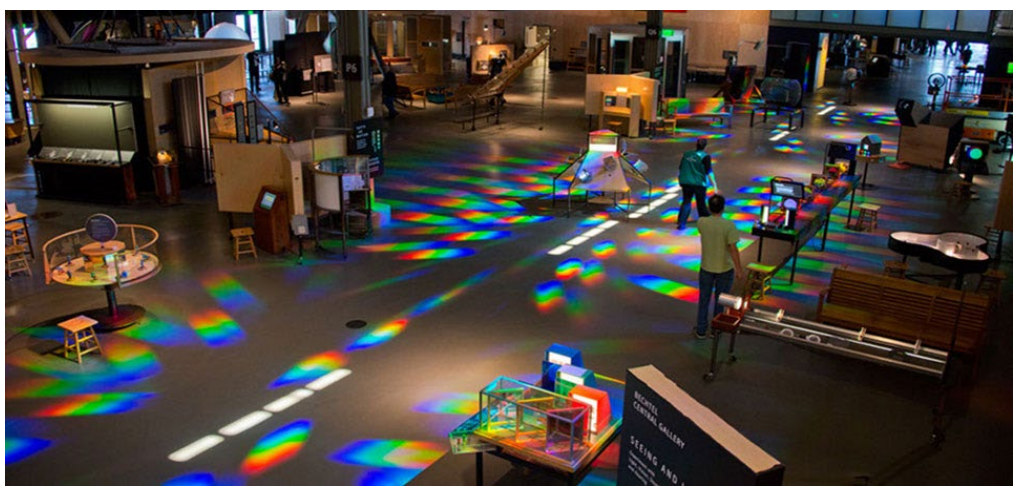




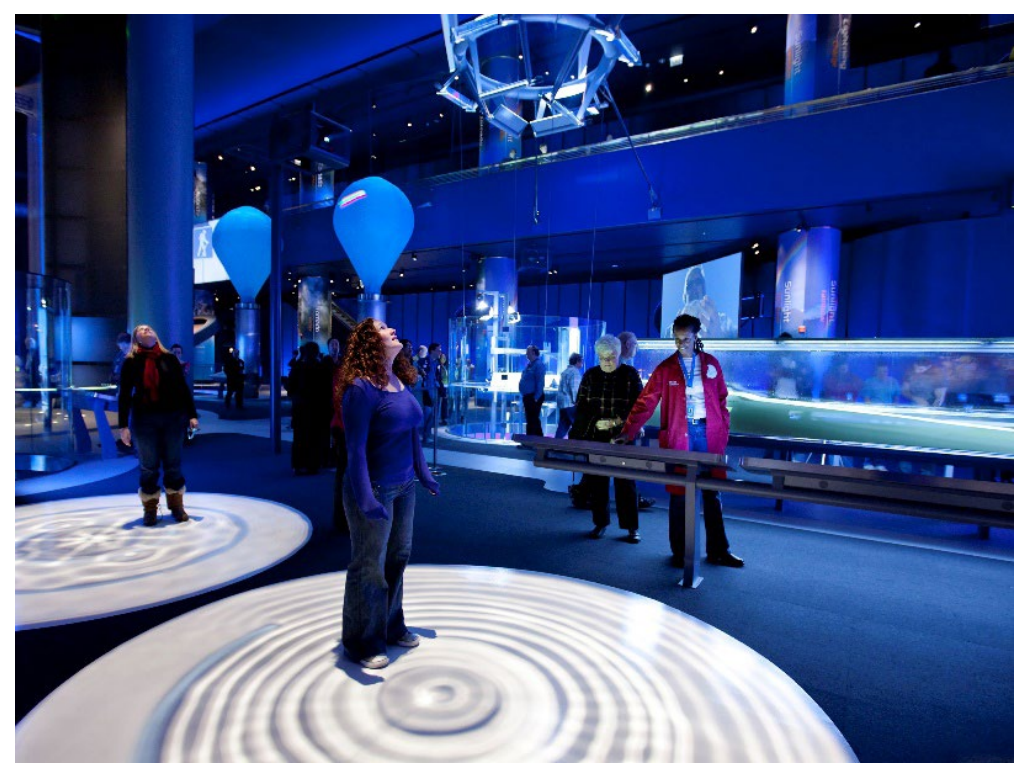

Figura 5. Exhibición "Science Storms". Museo de Ciencia e Industria de Chicago. Fuente: https://www.msichicago.org/press/exhibits-and-events/science-storms/.

Esta misma estrategia es utilizada en el Parque Explora en Medellín, Colombia, pero a diferencia de los dos anteriores no es un espacio reciclado sino un edificio de nueva planta diseñado por Alejandro Echeverri en 2004 y construido entre 2005 y 2008. Otra diferencia importante es que no se concibió como una única nave y un espacio global sino que los espacios expositivos se dispusieron en 4 grandes cajas, cada una de las cuales puede albergar una experiencia diferente, permitiendo así la transformación temática de las exposiciones. A pesar de esto, el espacio interior de cada caja le debe mucho a la de los museos anteriormente mencionados porque su concepción espacial es igualmente la de un gran espacio a dos niveles y la disposición de los artefactos y experiencias está también involucrada dentro de la circulación de los visitantes (Figura 6). 


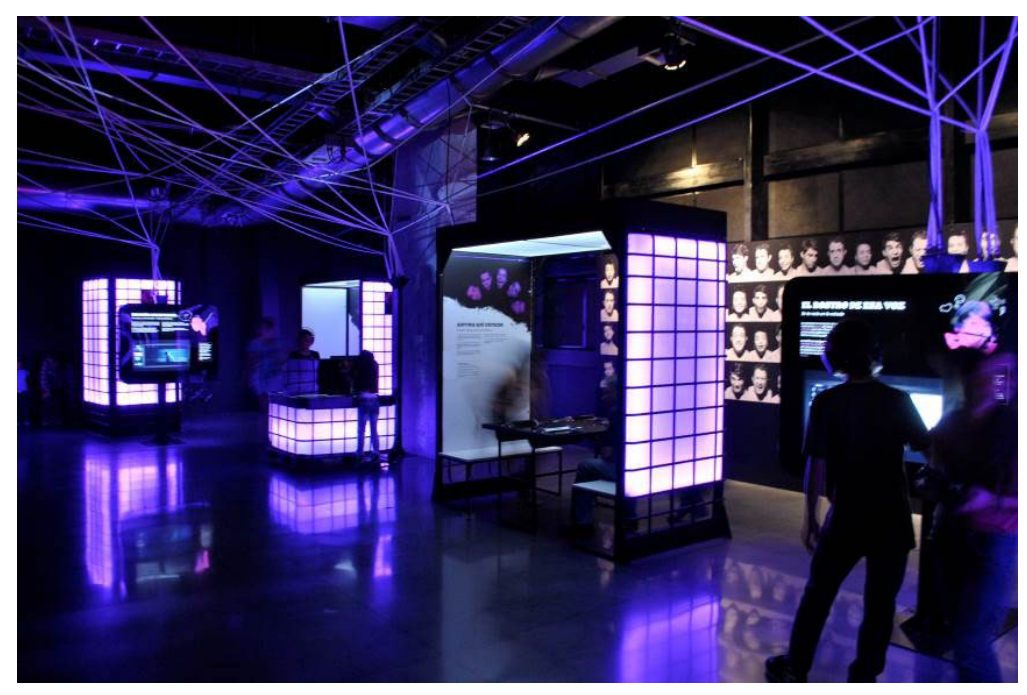

Figura 6. Sala de la mente. Parque Explora Medellín. Fuente: fotografía realizada por los autores, marzo de 2012

En síntesis, las particularidades de este espacio expositivo - y siguiendo con la línea de análisis tríadica- puede decirse que distingue al contenido científico como un saber demostrable y experimentable; en donde el artefacto expositivo es una representación para verificar los resultados científicos, cambiando en activa la experiencia del público. Estas características apuntan en varios sentidos a la analogía hecha al espacio expositivo como una tienda por departamentos donde reina la estrategia de seducir al usuario a usar el artefacto, a consumir el conocimiento que tiene para ofrecer. "Lejos de circunscribirse a las relaciones interpersonales, la seducción se ha convertido en el proceso general que tiende a regular el consumo, las organizaciones, la información, la educación, las costumbres" (Lipovetsky, 1986, p.17). 


\section{El estudio creativo: un espacio para la creación crítica. El medio: la provocación. El mensaje: ¡ya sé cómo conocer!}

Reconociendo de antemano los grandes aportes hechos a la comunicación de la ciencia desde los dos anteriores espacios expositivos, aún se persiste en perfeccionar la relación dada entre público y ciencia. Su pretensión es superar un modelo informativo y orientarse hacia un modelo que permita más bien la interacción comunicativa de doble vía entre público y ciencia, proyectando como consecuencia un nuevo espacio expositivo en el que se suscite la participación democrática con la ciencia.

Con esta intención en la mira, emerge a finales del siglo XX el modelo democrático (Durant, 1999) como una propuesta que responde a esa necesidad de cambio. Dicho modelo distingue al conocimiento científico como dimensión cultural asumida desde un punto histórico, parcial y provisional que convive con otros saberes igualmente válidos. Su énfasis no se dirige en "traducir el conocimiento científico" a un público no experto sino en lograr una comunicación bidireccional entre agentes sociales a los que se les reconoce un saber y experticias previos (Jiménez y Palacio, 2010). Es por ello que este nuevo espacio expositivo reclama que no solo sea comprendido como un lugar de divulgación del saber científico sino más bien como uno que logra involucrar verdaderamente al público en la cultura científica, lo que supone para el MC mudarse de la acción divulgativa —si se delimita su comprensión como la entrega de información desde una sola vía promueve la participación pública.

Esa transición participativa ha generado un nuevo campo de estudio de la comunicación de las ciencias denominado Public Engagement with Science

\footnotetext{
${ }^{6}$ Mónica Lozano (2005) referencia diferentes autores para distinguir que el fin de la divulgación reside en la transmisión de informaciones científicas y tecnológicas con un lenguaje accesible y decodificado, pero sosteniendo siempre una fidelidad con el contenido. Esta definición deja en claro que la pretensión es de orden informativo.
} 
and Technology (PEST) que algunos académicos distinguen como una evolución del modelo Public Understanding of Science (PUS) y otros como un modelo que tiene una naturaleza diferente a este debido a que no se concentra en analizar la percepción que el público tiene sobre la ciencia sino que más bien comprende diversos modos de participación e interacción para formar vínculos emocionales, sociales y políticos entre público y ciencia (Davies, 2013).

De modo que desengancharse de comunicar el resultado para provocar nuevas relaciones entre ciencia y sociedad es un giro que discurre hacia nuevos temas a exponer y que desemboca en un nuevo espacio expositivo. Para consolidar esta propuesta Erminia Pedretti (2002) cuestiona las exposiciones centradas en la comunicación de fenómenos científicos dado que en ellas se presenta una información precisa, delimitada, monolítica y apolítica de la ciencia y que por lo tanto no se permite el cuestionamiento de tales contenidos. Es por ello que Pedretti distingue a la exposición crítica como un nuevo tipo expositivo puesto que inspira preguntas sobre la ciencia y su fiabilidad, reconoce la provisionalidad del conocimiento científico y considera a la ciencia como una actividad humana y social; es decir que es una exposición que "habla sobre los procesos de la ciencia, su naturaleza y sobre el contexto socio-cultural de la ciencia y la tecnología" (Pedretti, 2002, p. 9)

Este tipo expositivo crítico se acerca a las particularidades comunicativas propuestas en el modelo democrático y el modelo PEST. En este se permite no solo la participación del público sino que, además, supone una comunicación del conocimiento científico desde una perspectiva social y no desde el fenómeno. En consecuencia, el espacio expositivo bajo dichas características distingue que el contenido científico ya no sea presentado como un hallazgo o teoría a informar sino más bien como una forma más de conocimiento que

\footnotetext{
${ }^{7}$ Esta categorización expositiva de Pedretti (2002) emerge de los dos tipos expositivos descritos por Wellington (1998): la pedagógica que tiene la función de enseñar/describir el fenómeno y la experiencial que permite la experimentación del fenómeno; exposiciones que pueden corresponderse con la vía descriptiva y la demostrativa respectivamente.
} 
es provisional, parcial y completamente dinámico, y que posee una relación profunda con la sociedad; de ahí que los asuntos que la exposición debe abordar sean temas que permitan una discusión entre ciencia y sociedad, explícitamente de carácter controversial para estas dos entidades. No es posible hacer una reflexión crítica sobre un hecho o fenómeno incuestionable, sobre un tema de controversia socio-científica si lo es.

Ciertamente, si el asunto expositivo es de carácter controversial, la experiencia deberá provocar el discernimiento crítico del público frente al contenido presentado. Moverse de la concepción del visitante como un autómata que interpreta el conocimiento a través de la manipulación de máquinas hacia una provocación crítica supone que este pueda cuestionar, divagar, intuir, dudar, especular, formular y argumentar sus reflexiones frente a los asuntos de controversia socio-científica. Con base en lo anterior, el contenido y la experiencia de la exposición crítica distinguen que el visitante pasa del "ya conozco" al "ya sé cómo conocer"; explícitamente pasa de la adquisición de información a la formación de habilidades para conocer (Bradburne, 1998). Esta circunstancia, dice Pedretti (2002):

refleja un giro radical de las tradicionales exposiciones interactivas o hands-on, y su preocupación por la experiencia sensorial inmediata y la explicación científica del fenómeno. Es una especie de revolución Kuhniana, pues reta a los museos y centros de ciencia a reconceptualizar su rol y propósito. (p. 10)

En consecuencia, las afirmaciones de Pedretti y Bradburne formulan que el cambio comunicativo dentro del MC radica en fomentar y promover en el público el uso del sentido crítico frente a los asuntos de controversia científica. En otras palabras, la preocupación del museo por provocar un pensamiento crítico —como también lo persiguen las instituciones educativas- es para salvaguardar al público de la adquisición acrítica del conocimiento (Lipman, 1997). Que el público salga del museo con la claridad del "ya sé cómo 
conocer" tiene que ver directamente con fomentar el "pensar bien"; en ese sentido la exposición crítica, además de presentar asuntos críticos de la ciencia, persigue que el público pueda discernir de manera clara y lógica para resolver problemas de manera creativa bajo el uso de criterios propicios del asunto expuesto.

Pero ¿cómo provocar el pensamiento crítico del público en el MC? Las primeras experiencias que ha acogido el MC para servir a este propósito han sido los espacios de creación autónoma que confieren una aproximación diferente a la ciencia y la tecnología al centrar sus prácticas en la construcción y fabricación de artefactos a través de la formulación de hazlo tú mismo o Do it yourself $(D / Y)$.

Escenarios alternativos como los Hackerspace, Makerspace y las Maker faire ${ }^{8}$ son laboratorios, talleres o ferias en los que se concentran recursos técnicos y de conocimiento en un solo lugar; en donde se permite la experimentación y la libre creación de artefactos ya sean para fines artísticos, tecnológicos o científicos. Estos escenarios son referentes interesantes para el MC puesto que valoran la exploración creativa como medio para abordar el conocimiento de otras áreas y porque permite la concreción de ideas alternativas frente a la tecnología y la ciencia a través de la experimentación y composición de artefactos. Aunque en la mayoría de los casos de la cultura Maker o DIY lo que cobra valor son los resultados artefactuales. No obstante, podría decirse que lo que es interesante para el MC no es el resultado sino su proceso de pensar a través del hacer ${ }^{9}$ : explícitamente las reflexiones, especulaciones y compresiones que dicha construcción suscita alrededor de su exploración creativa; de esta pretensión es que surge la analogía con el estudio creativo.

\footnotetext{
${ }^{8}$ Espacios colaborativos y de libre aprendizaje que fomentan la creación y/o manipulación de artefactos ya sean para fines artísticos, tecnológicos o científicos. Hacen parte del Maker Movement impulsados desde 2005 por la revista MAKE, y que convoca a la comunidad de "hazlo tú mismo" (Do lt Yourself) para la fabricación de artefactos tecnológicos.

${ }^{9}$ Este concepto establece que reflexión (mente) y acción (cuerpo) se integran para coproducir el aprendizaje y el razonamiento (Klemmer Hartmann and Takayama, 2006).
} 
Sin embargo es importante distinguir que las prácticas dadas dentro de este estudio creativo, aún no han sido conjugadas del todo con la acción expositiva del MC. Lo que sí ha sucedido hasta el momento es que varios MC están alojando a estos estudios creativos como laboratorios o talleres especializados como islas o apéndices. Una muestra de ello es el Design Lab del New York Hall of Science (Figura 7), que permite a los visitantes una relación más activa y cercana con la temática de construcción propuesta por el museo a través de espacios abiertos con delimitaciones bajas y mobiliario que posibilitan diversas posturas corporales; entretanto el modelo espacial se acerca más a un taller de artesano que a una sala de museo (Figura 8).

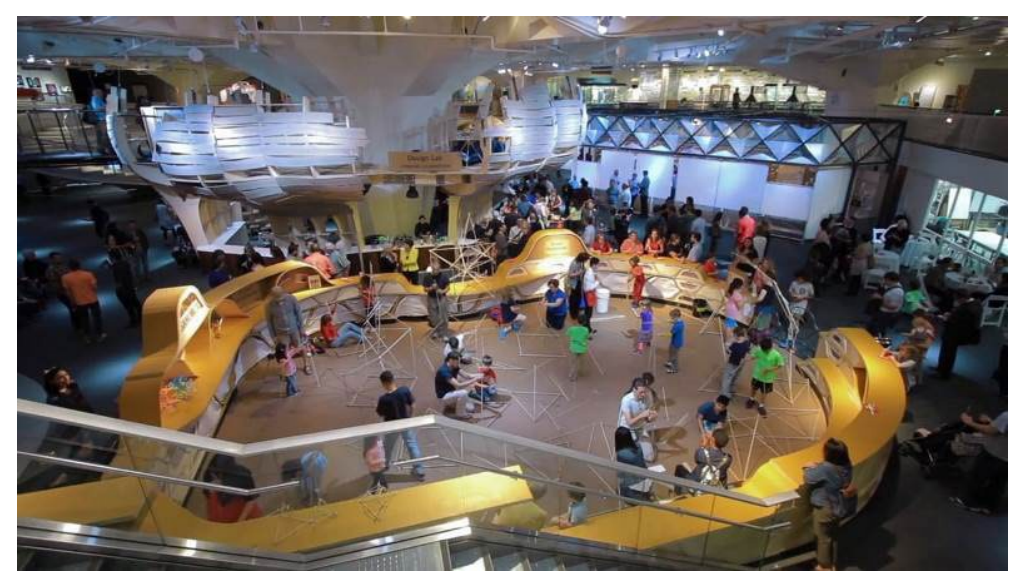

Figura 7. "Sandbox". Design Lab, New York Hall of Science. Fuente: http://i.vimeocdn.com/video/483237986_1280x720.jpg. 


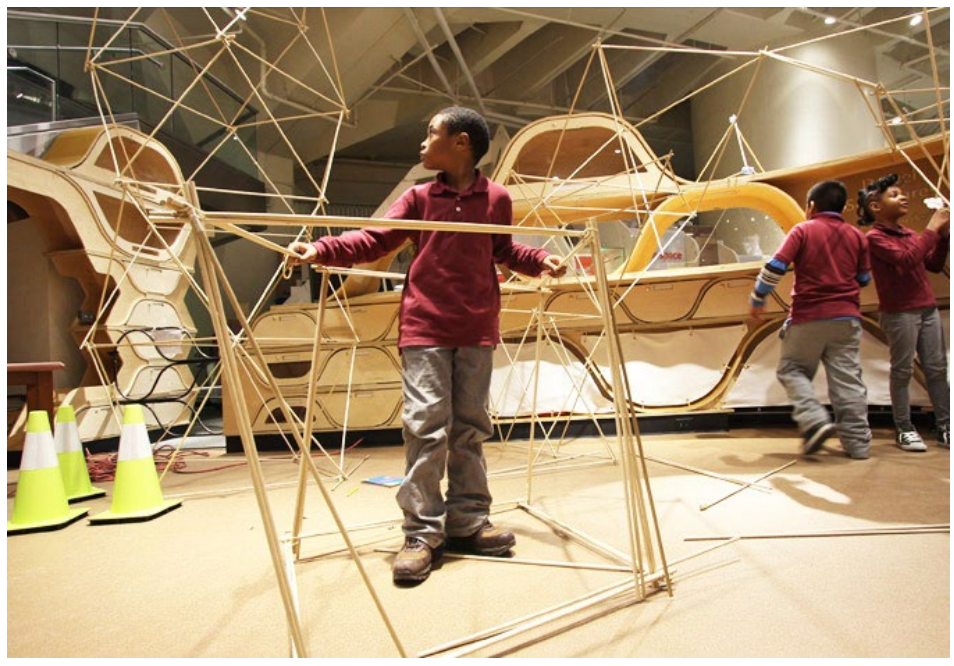

Figura 8. "Sandbox". Design Lab, New York Hall of Science. Fuente: http://www.situstudio.com/works/built/nysci-design-lab.

Ahora bien, ¿cómo hacer que la participación creativa del público se conjugue con el espacio expositivo? Inicialmente se podría pronosticar que no habría algún problema que estas dos actividades pudieran reunirse; es más, propone una potencia comunicativa y a la vez formativa para el MC contemporáneo. La participación creativa del público propone que el espacio expositivo deba ser concebido como inacabado, una idea tan interesante como polémica: pues al no haber total control por parte del diseño expositivo sobre los resultados variopintos de la participación del público, el aspecto final de la exposición es siempre inesperada y variable; a favor habrá que reconocer que esta condición de incertidumbre es una de las características que mejor describen a la sociedad contemporánea o posmoderna, dotando así a estos espacios de un espíritu de su tiempo bastante fuerte. Instar que en este tipo de espacio expositivo 
el público use su conocimiento previo para participar en asuntos críticos de la ciencia, proponer soluciones creativas y que su propuesta construida haga parte de la exposición, transforma al museo en un estudio creativo, al visitante en un creador crítico y al diseño expositivo en un provocador crítico.

\section{Conclusión}

Este análisis buscó evidenciar que el espacio expositivo es un actor fundamental en la comunicación de la ciencia dentro del museo y que en los tres espacios expositivos definidos hay que reconocer el valor que cada uno tiene: el primero y el segundo, aunque expresen mensajes diferentes, proponen un acercamiento pasivo al conocimiento expuesto, asunto importante para generar conceptos previos sobre el tema; el tercero, propone poner en crisis ese conocimiento adquirido. Esto distingue que los tres espacios y las tres funciones que cada uno plantea son todas ellas necesarias para la adquisición, comprensión y análisis del conocimiento científico en busca de fomentar la participación del público en la cultura científica desde en el museo de ciencias.

Es así que las anteriores metamorfosis presentadas, aunque le deban su naturaleza comunicativa al momento histórico en el que emergen y por lo que pareciera que cada espacio fuese un sucesor del anterior, cada una dispone un aporte relevante para la interacción del público con la ciencia. El mensaje o acción comunicativa que cada una expresa con su comprensión del contenido, artefactos y experiencia, contribuye a una porción importante de la experiencia de conocimiento de la ciencia; razón por la cual los mensajes deban operar en el museo de una forma más simbiótica que dispersa. En consecuencia, la metamorfosis más que ser una transformación eliminatoria, es una transformación que incrementa el potencial del museo para la comunicación de las ciencias y la democratización del conocimiento. No obstante, es preciso señalar que la exposición del museo mismo como un estudio creativo tiene 
pendiente profundizar en actividades tanto prácticas como investigativas desde las que se pueda prototipar y observar las características con las que se lo describió y que permitan advertir la experiencia y los efectos que dicha configuración espacial y comunicativa tiene en el público, en el museo, en los científicos y en la ciencia.

\section{Referencias}

Bellido, M.L. (2001). Arte, museos y nuevas tecnologías. Asturias, España: Ediciones Trea S.L.

Bradburne, J. (1990). Beyond Hands-On: Truth-telling and the Doing of Science. En R. Glanville and G. de Zeeuw (Eds.), Mutual Uses of Science and Cybernetics (pp. 53-61). Amsterdam, Netherlands: Thesis Publishers.

Bradburne, J. (1998). Dinosaurs and white elephants: The science center in the 21st century. Museum Management and Curatorship, 17 (2), 119-137.

Davies, S. (2013). Constituting Public Engagement: Meanings and Genealogies of PEST in Two U.K. Studies. Science Communication, 35 (6), 687-707.

Durant, J. (1999). Participatory technology assessment and the democratic model of the public understanding of science. Science and Public Policy, 26 (5), 313-319.

Franco-Avellaneda, M. (2013). Museos, artefactos y sociedad: ¿cómo se configura su dimensión educativa? Universitas Humanística, 76, 97-123.

Hernández, F. y Rubio, X. (2009). Interactividad didáctica y museos. Enseñanza de las Ciencias Sociales, 8, 91-96.

Jiménez, S. y Palacio, M. (2010). Comunicación de la ciencia y la tecnología en museos y centros interactivos de la ciudad de Medellín. Universitas Humanística, 69, 227-257. 
Klemmer, S.R., Hartmann, B. and Takayama, L. (2006). How bodies matters: Five themes for interaction design (pp. 140-149). En AMC, DIS '06 Proceedings of the 6th conference. New York, USA: ACM Digital Library.

Labandeira, S. (2008). Breve recorrido por la evolución del concepto museo. Revista Museo, 13, 320-325.

Lipman, M. (1997). Pensamiento complejo y educación. Madrid, España: Ediciones de la Torre.

Lipovetsky, G. (1986). La era del vacío: ensayos sobre el individualismo contemporáneo. Barcelona, España: Anagrama.

Lozano, M. (2005). Programas y experiencias en popularización de la ciencia y la tecnología. Panorámica desde los países del Convenio Andrés Bello. Bogotá, Colombia: Convenio Andrés Bello.

McLuhan, M. (1996). Comprender los medios de comunicación: las extensiones del ser humano. Barcelona, España: Paidós.

McManus, P. (1992). Topics in Museums and Science Education. Studies in Science Education, 20 (1), 157-182.

Nussbaum, M. (2010). Sin fines de lucro. Por qué la democracia necesita de las humanidades. Madrid, España: Katz.

Oppenheimer, F. (1968). Rationale for a Science Museum. Curator: The Museum Journal, 1 (3), 206-209.

Orozco, G. (2005). Los museos interactivos como mediadores pedagógicos. Sinéctica, Revista Electrónica de Educación, 26, 38-50.

Pastor, I. (2011). Pedagogía museística. Nuevas perspectivas y tendencias actuales. Barcelona, España: Ariel. 
Pedretti, E. (2002). T. Kuhn Meets T. Rex: Critical Conversations and New Directions in Science Centers and Science Museums. Studies in Science Education, 37 (1), 1-41.

Poulot, D. (2011). Museo y museología. Madrid, España: Abada Editores.

Rennie, L. (2007). Learning science outside of school. En S.K. Abell and N.G. Lederman (Eds.), Handbook of Research on Science Education. New Jersey, USA: Routledge.

Rousseau, J.-J. (1982). Emilio. Madrid, España: EDAF.

Vélez, C. (2013). De los ojos a las manos: tocar el espacio. El espacio táctil en la arquitectura moderna. Medellín, Colombia: Universidad Nacional de Colombia.

Wellington, J.J. (1998). Interactive science centers and science education. Croner's Heads of Science Bulletin, 16.

Como citar: Pérez-Orrego, N. y Arango, J. (2019). Metamorfosis del espacio expositivo en el museo de ciencias: de cueva de tesoros a estudio creativo. Revista KEPES, 16 (19), 39-63. DOI: 10.17151/ kepes.2019.16.19.3 